\title{
Metric Properties of Semialgebraic Mappings
}

\author{
Krzysztof Kurdyka $^{1} \cdot$ Stanisław Spodzieja $^{2}$ (D) \\ Anna Szlachcińska ${ }^{2}$
}

Received: 21 February 2013 / Revised: 26 February 2016 / Accepted: 9 March 2016 /

Published online: 21 March 2016

(C) The Author(s) 2016. This article is published with open access at Springerlink.com

\begin{abstract}
We give various quantitative versions of Łojasiewicz inequalities for semialgebraic sets and mappings, both in the local and global case.
\end{abstract}

Keywords Łojasiewicz exponent - Semialgebraic set - Semialgebraic mapping · Polynomial mapping

Mathematics Subject Classification 14P20 $\cdot 14 \mathrm{P} 10 \cdot 32 \mathrm{C} 07$

\section{Introduction}

Łojasiewicz inequalities emerged in the late 1950s as the main tool in the division of distributions by a real polynomial (Hörmander [17]) and by a real analytic function (Łojasiewicz [25,26]). Since then they have turned out to be of use in numerous branches of mathematics, including differential equations, dynamical systems and

Editor in Charge: Günter M. Ziegler

Krzysztof Kurdyka

Krzysztof.Kurdyka@univ-savoie.fr

Stanisław Spodzieja

spodziej@math.uni.lodz.pl

Anna Szlachcińska

anna_loch@wp.pl

1 Laboratoire de Mathematiques (LAMA), Universié Savoie Mont Blanc, UMR-5127 de CNRS, 73-376, Le Bourget-du-Lac Cedex, France

2 Faculty of Mathematics and Computer Science, University of Łódź, S. Banacha 22, 90-238 Lodz, Poland 
singularity theory (see for instance $[24,28,38]$ ). Quantitative versions of these inequalities, involving e.g. computing or estimating the relevant exponents, are of importance in real and complex algebraic geometry (see [43] and also [31-33]). Recently a strong demand for explicit estimates of the Łojasiewicz exponent comes from optimization theory (see for instance [23,37]) and also from estimates for global error bounds [27].

Our goal is to give various quantitative versions of these inequalities in the real case both in the local and global context. We denote by $\mathbb{K}$ the field $\mathbb{R}$ of real numbers or the field $\mathbb{C}$ of complex numbers.

Let $X \subset \mathbb{K}^{N}$ be a closed semialgebraic set. (If $\mathbb{K}=\mathbb{C}$ we consider $X$ as a subset of $\mathbb{R}^{2 N}$.) Assume that $0 \in X$ is an accumulation point of $X$ and $f, g: X \rightarrow \mathbb{R}$ are two continuous semialgebraic functions such that $f^{-1}(0) \subset g^{-1}(0)$. Then there are positive constants $C, \eta, \varepsilon$ such that the following Łojasiewicz inequality holds (see e.g. [4]):

$$
|f(x)| \geq C|g(x)|^{\eta} \quad \text { if } \quad x \in X,|x|<\varepsilon .
$$

The infimum of the exponents $\eta$ in (1.1) is called the Eojasiewicz exponent of the pair $(f, g)$ on the set $X$ at 0 and is denoted by $\mathcal{L}_{0}(f, g \mid X)$. It is known (see [3]) that $\mathcal{L}_{0}(f, g \mid X)$ is a rational number; moreover, inequality (1.1) holds actually with $\eta=\mathcal{L}_{0}(f, g \mid X)$ for some $\varepsilon, C>0$ (see [41]). An asymptotic estimate for $\mathcal{L}_{0}(f, g \mid X)$ was obtained by Solernó [39]; we shall discuss it in Remark 2.4. Inequality (1.1) is valid in a more general setting of functions definable in an o-minimal polynomially bounded structure (in particular for subanalytic functions) (see [12,16]).

From the point of view of applications the most interesting case of inequality (1.1) is when $f$ is a semialgebraic function and $g(x)=\operatorname{dist}\left(x, X \cap f^{-1}(0)\right)$. We shall consider the distance induced by the Euclidean norm. By convention $\operatorname{dist}(x, \emptyset)=1$. More precisely, we shall consider the following case. Let $F=\left(f_{1}, \ldots, f_{m}\right): \mathbb{K}^{N} \rightarrow \mathbb{K}^{m}$ be a semialgebraic mapping and $X \subset \mathbb{K}^{N}$ a closed semialgebraic set such that $0 \in X$ is an accumulation point of $X$. So we have the following Łojasiewicz inequality:

$$
|F(x)| \geq C \operatorname{dist}\left(x, F^{-1}(0) \cap X\right)^{\eta} \quad \text { if } x \in X,|x|<\varepsilon .
$$

The smallest exponent $\eta$ in (1.2) is called the Łojasiewicz exponent of $F$ on the set $X$ at 0 and is denoted by $\mathcal{L}_{0}^{\mathbb{K}}(F \mid X)$. In Sect. 2 we shall give explicit bounds for $\mathcal{L}_{0}^{\mathbb{K}}(F \mid X)$ in terms of the degrees of the data involved. The main result of this section is an explicit estimate for the local Łojasiewicz exponent for separation of semialgebraic sets (see Theorem 1.1).

The second aim of this article is to obtain similar results but for the Łojasiewicz exponent at infinity. Assume now that a closed semialgebraic set $X \subset \mathbb{K}^{N}$ is unbounded. By the Eojasiewicz exponent at infinity of a mapping $F: X \rightarrow \mathbb{K}^{m}$ we mean the supremum of the exponents $v$ in the following Łojasiewicz inequality:

$$
|F(x)| \geq C|x|^{\nu} \quad \text { for } \quad x \in X,|x| \geq R
$$

for some positive constants $C, R$; we denote it by $\mathcal{L}_{\infty}^{\mathbb{K}}(F \mid X)$. If $X=\mathbb{K}^{N}$ we call the exponent $\mathcal{L}_{\infty}^{\mathbb{K}}(F \mid X)$ the Eojasiewicz exponent at infinity of $F$ and denote it by 
$\mathcal{L}_{\infty}^{\mathbb{K}}(F)$. Clearly $\mathcal{L}_{\infty}^{\mathbb{K}}(F \mid X)$ may be negative. Note that inequality (1.3) holds only when $X \cap F^{-1}(0)$ is compact.

The next inequality, called the Hörmander-Łojasiewicz inequality [17], is always valid for a continuous semialgebraic mapping:

$$
|F(x)| \geq C\left(\frac{\operatorname{dist}\left(x, F^{-1}(0) \cap X\right)}{1+|x|^{2}}\right)^{\theta} \text { for } x \in X,
$$

where $C, \theta$ are some positive constants. In Sect. 3 we state Theorem 3.2 which is a global quantitative version of regular separation at infinity of semialgebraic sets. It implies, in particular, an estimate for the exponent (see Corollaries 3.3, 3.4).

The paper is organized as follows: in Sects. 2 and 3 we discuss Łojasiewicz inequalities respectively in the local and global case. The proofs of the main results are given in the last section.

\section{The Lojasiewicz Exponent at a Point}

We will give an estimate from above of the Łojasiewicz exponent for the regular separation of closed semialgebraic sets and for a continuous semialgebraic mapping on a closed semialgebraic set. Let us start from some notation. Let $X \subset \mathbb{R}^{N}$ be a closed semialgebraic set. It is known that $X$ has a decomposition

$$
X=X_{1} \cup \cdots \cup X_{k}
$$

into the union of closed basic semialgebraic sets

$$
X_{i}=\left\{x \in \mathbb{R}^{N}: g_{i, 1}(x) \geq 0, \ldots, g_{i, r_{i}}(x) \geq 0, h_{i, 1}(x)=\cdots=h_{i, l_{i}}(x)=0\right\},
$$

$i=1, \ldots k$ (see [4]), where $g_{i, 1}, \ldots, g_{i, r_{i}}, h_{i, 1}, \ldots, h_{i, l_{i}} \in \mathbb{R}\left[x_{1}, \ldots, x_{N}\right]$. Assume that $r_{i}$ is the smallest possible number of the inequalities $g_{i, j}(x) \geq 0$ in the definition of $X_{i}$, for $i=1, \ldots, k$. Denote by $r(X)$ the minimum of $\max \left\{r_{1}, \ldots, r_{k}\right\}$ over all decompositions (2.1) into unions of sets of the form (2.2). As shown by Bröcker [6] (cf. $[5,35])$,

$$
r(X) \leq \frac{N(N+1)}{2}
$$

Denote by $\kappa(X)$ the mimimum of the numbers

$$
\max \left\{\operatorname{deg} g_{1,1}, \ldots, \operatorname{deg} g_{k, r_{k}}, \operatorname{deg} h_{1,1}, \ldots, \operatorname{deg} h_{k, l_{k}}\right\}
$$

over all decompositions (2.1) of $X$ into the union of sets of the form (2.2), provided $r_{i} \leq r(X)$. Obviously $r(X)=0$ if and only if $X$ is an algebraic set. The numbers $r(X)$ and $\kappa(X)$ characterize the complexity of the semialgebraic set $X$. For more information about the complexity see for example [2,4,34]. 
Theorem 2.1 Let $X, Y \subset \mathbb{R}^{N}$ be closed semialgebraic sets, and suppose $0 \in X \cap Y$. Set $r=r(X)+r(Y)$ and $d=\max \{\kappa(X), \kappa(Y)\}$. Then there exist a neighbourhood $U \subset \mathbb{R}^{N}$ of 0 and a positive constant $C$ such that

$$
\operatorname{dist}(x, X)+\operatorname{dist}(x, Y) \geq C \operatorname{dist}(x, X \cap Y)^{d(6 d-3)^{N+r-1}} \quad \text { for } x \in U .
$$

If, additionally, 0 is an isolated point of $X \cap Y$, then for some neighbourhood $U \subset \mathbb{R}^{N}$ of 0 and some positive constant $C$,

$$
\operatorname{dist}(x, X)+\operatorname{dist}(x, Y) \geq C|x|^{\frac{(2 d-1)^{N+r}+1}{2}} \text { for } x \in U
$$

The proof of the above theorem will be carried out in Sect. 4. The key point in the proof will be the following inequality [22, Cor. 8]. Let $X=\left(g_{1}, \ldots, g_{k}\right)^{-1}(0)$ and $Y=\left(h_{1}, \ldots, h_{l}\right)^{-1}(0) \subsetneq \mathbb{R}^{N}$, where $g_{i}, h_{j} \in \mathbb{R}\left[x_{1}, \ldots, x_{N}\right]$ are polynomials of degree not greater than $d$. Let $a \in \mathbb{R}^{N}$. Then there exists a positive constant $C$ such that

$$
\operatorname{dist}(x, X)+\operatorname{dist}(x, Y) \geq C \operatorname{dist}(x, X \cap Y)^{d(6 d-3)^{N-1}}
$$

in a neighbourhood of $a$. If, additionally, $a$ is an isolated point of $X \cap Y$, then

$$
\operatorname{dist}(x, X)+\operatorname{dist}(x, Y) \geq C|x-a|^{\frac{(2 d-1)^{N}+1}{2}}
$$

in a neighbourhood of $a$ for some positive $C>0$, which is a consequence of [14].

Theorem 2.1 implies

Corollary 2.2 Let $F: X \rightarrow \mathbb{R}^{m}$ be a continuous semialgebraic mapping, where $X \subset \mathbb{R}^{N}$ is a closed semialgebraic set, and suppose $0 \in X$ and $F(0)=0$. Set $r=r(X)+r(\operatorname{graph} F)$ and $d=\max \{\kappa(X), \kappa(\operatorname{graph} F)\}$. Then

$$
\mathcal{L}_{0}^{\mathbb{R}}(F \mid X) \leq d(6 d-3)^{N+r-1}
$$

If, additionally, 0 is an isolated zero of $F$, then

$$
\mathcal{L}_{0}^{\mathbb{R}}(F \mid X) \leq \frac{(2 d-1)^{N+r}+1}{2} .
$$

Remark 2.3 The inequality (2.6) is crucial for estimating the rate of convergence of algorithms (based on semi-definite programming) of minimization of a polynomial on a basic semialgebraic set. Indeed, (2.6) enabled us [23] to reduce effectively the problem of minimizing polynomials on a compact semialgebraic set to the case of minimizing polynomials on a ball, which is much simpler [36].

Remark 2.4 We shall now comment on the result of Solernó [39] concerning the Łojasiewicz exponent $\mathcal{L}_{0}(f, g \mid X)$ in the inequality (1.1) for a pair $(f, g)$ of continuous 
semialgebraic functions on a closed semialgebraic set $X \subset \mathbb{R}^{N}$. In general his estimate is of the form

$$
\mathcal{L}_{0}(f, g \mid X) \leq D^{M^{c a}}
$$

where $D$ is a bound for the degrees of the polynomials involved in a description of $f$, $g$ and $X ; M$ is the number of variables in these formulas (so in general $M \geq N$ ); $a$ is the maximum number of alternating blocs of quantifiers in these formulas; and $c$ is an (unspecified) universal constant. The estimate $\left(S_{a}\right)$ was obtained from the effective Tarski-Seidenberg theorem [15].

In our Corollary 2.2 only the function $g(x)=\operatorname{dist}\left(x, X \cap F^{-1}(0)\right)$ is defined by a formula which is not quantifier-free, and it has two alternating blocs of quantifiers, hence $a=2$. So Solernó's estimate $\left(\mathrm{S}_{a}\right)$ reads $\mathcal{L}_{0}^{\mathbb{R}}(F \mid X) \leq d^{(N+2)^{2 c}}$, which is comparable with our estimate $\mathcal{L}_{0}^{\mathbb{R}}(F \mid X) \leq d(6 d-3)^{N+r-1}$ since $r(X) \leq \frac{1}{2} N(N+1)$ by (2.3). Indeed, we believe that the universal constant $c$ is at least 1 , probably $c \gg 1$. Needless to say, our estimate is explicit.

Recall that for a real polynomial mapping $F: \mathbb{R}^{N} \rightarrow \mathbb{R}^{m}$ such that $d=\operatorname{deg} F$ (where $\operatorname{deg} F$ is the maximum of the degrees of the components of $F$ ) we have

$$
\mathcal{L}_{0}^{\mathbb{R}}(F) \leq d(6 d-3)^{N-1}
$$

(see [22, Cor. 6] or [29]). Actually both papers are based on an estimate for the Łojasiewicz exponent in the gradient inequality obtained in [11,13].

We now consider a polynomial mapping restricted to an algebraic set. From Corollary 2.2 we obtain an estimation of its local Łojasiewicz exponent, also for a non-isolated zero-set (cf. [30,40] for mappings with isolated zeros).

Corollary 2.5 Let $F:\left(\mathbb{K}^{N}, 0\right) \rightarrow\left(\mathbb{K}^{m}, 0\right)$ be a polynomial mapping, let $X \subset \mathbb{K}^{N}$ be an algebraic set defined by a system of equations $g_{1}(x)=\cdots=g_{r}(x)=0$, where $g_{1}, \ldots, g_{r} \in \mathbb{K}\left[x_{1}, \ldots, x_{N}\right]$, and let $d=\max \left\{\operatorname{deg} F, \operatorname{deg} g_{1}, \ldots, \operatorname{deg} g_{r}\right\}$. Assume that $d>0$ and $0 \in X$.

(a) If $\mathbb{K}=\mathbb{R}$, then $\mathcal{L}_{0}^{\mathbb{R}}(F \mid X) \leq d(6 d-3)^{N-1}$.

(b) If $\mathbb{K}=\mathbb{C}$, then $\mathcal{L}_{0}^{\mathbb{C}}(F \mid X) \leq d^{N}$.

Indeed, assertion (a) immediately follows from Corollary 2.2. We will prove (b). Let $G=\left(F, g_{1}, \ldots, g_{r}\right): \mathbb{C}^{N} \rightarrow \mathbb{C}^{m+r}$. We can assume that $m \geq N$. Similarly to [42, Thm. 1], we prove that there exists a linear mapping $L=\left(L_{1}, \ldots, L_{m}\right): \mathbb{C}^{m+r} \rightarrow$ $\mathbb{C}^{m}$ of the form $L_{i}\left(y_{1}, \ldots, y_{m}\right)=y_{i}+\sum_{j=r+1}^{m} \alpha_{i, j} y_{j}, i=1, \ldots, m$, where $\alpha_{i, j} \in \mathbb{C}$, such that $\mathcal{L}_{0}^{\mathbb{C}}(G \mid X)=\mathcal{L}_{0}^{\mathbb{C}}(L \circ G \mid X)$. Moreover, deg $L_{j} \circ G \leq d$ for $j=1, \ldots, m$. Cygan [8] proved that for analytic sets $Z, Y \subset \mathbb{C}^{N+m}$ the intersection index at 0 of $Z$ and $Y$ is a separation exponent of $Z$ and $Y$ at $0 \in Z \cap Y$. It is known that for $Z=\mathbb{C}^{N} \times\{0\}$ and $Y=\operatorname{graph} L \circ G$, the index does not exceed $d^{N}$ (see $[10,44]$ ), so $\mathcal{L}_{0}^{\mathbb{C}}(L \circ G) \leq d^{N}$. Since $G^{-1}(0)=F^{-1}(0) \cap X$ and by definition of $L$ we have $G(x)=(F(x), 0)$ for $x \in X$, it follows that $\mathcal{L}_{0}^{\mathbb{C}}(F \mid X) \leq d^{N}$, proving (b). 


\section{The Lojasiewicz Exponent at Infinity}

Let us first recall some known results on the Łojasiewicz exponent at infinity of a polynomial mapping $F=\left(f_{1}, \ldots, f_{m}\right): \mathbb{C}^{N} \rightarrow \mathbb{C}^{m}$ on an algebraic set $V \subset \mathbb{C}^{N}$ (see definition of $\mathcal{L}_{\infty}^{\mathbb{C}}(F \mid V)$ and $\mathcal{L}_{\infty}^{\mathbb{C}}(F)$ in Introduction). Let deg $f_{j}=d_{j}, j=1, \ldots, m$, $d_{1} \geq \cdots \geq d_{m}>0$ and set

$$
B\left(d_{1}, \ldots, d_{m} ; k\right)= \begin{cases}d_{1} \cdots d_{m} & \text { for } m \leq k \\ d_{1} \cdots d_{k-1} d_{m} & \text { for } m>k\end{cases}
$$

Chạdzyński [7] proved that

$$
\mathcal{L}_{\infty}^{\mathbb{C}}(F) \geq d_{2}-d_{1} d_{2}+\sum_{b \in F^{-1}(0)} \mu_{b}(F)
$$

where $\mu_{b}(F)$ is the multiplicity of $F$ at $b$, provided $N=m=2$ and $\# F^{-1}(0)<\infty$. For arbitrary $m \geq N$, under the assumption $\# F^{-1}(0)<\infty$, Kollár [21] proved that

$$
\mathcal{L}_{\infty}^{\mathbb{C}}(F) \geq d_{m}-B\left(d_{1}, \ldots, d_{m} ; N\right)
$$

then Cygan et al. [10] improved this to

$$
\mathcal{L}_{\infty}^{\mathbb{C}}(F) \geq d_{m}-B\left(d_{1}, \ldots, d_{m} ; N\right)+\sum_{b \in F^{-1}(0)} \mu_{b}(F)
$$

where $\mu_{b}(F)$ is the intersection multiplicity (in the sense of Achilles et al. [1]) of the graph of $F$ and $\mathbb{C}^{n} \times\{0\}$ at the point $(b, 0)$. For a complex $k$-dimensional algebraic variety $V \subset \mathbb{C}^{N}$ of degree $D$ the following estimate was obtained by Jelonek $[18,19]$ :

$$
\mathcal{L}_{\infty}^{\mathbb{C}}(F \mid V) \geq d_{m}-D \cdot B\left(d_{1}, \ldots, d_{m} ; k\right)+\sum_{b \in F^{-1}(0) \cap V} \mu_{b}(F),
$$

where $\#\left(F^{-1}(0) \cap V\right)<\infty$. Cygan [9] gave the following global inequality:

$$
|F(x)| \geq C\left(\frac{\operatorname{dist}\left(x, F^{-1}(0)\right)}{1+|x|^{2}}\right)^{B\left(d_{1}, \ldots, d_{m} ; N\right)} \text { for } \quad x \in \mathbb{C}^{N}
$$

for some positive constant $C$. Moreover she proved in [8] that for complex algebraic sets $X, Y \subset \mathbb{C}^{N}$ there exists a positive constant $C$ such that

$$
\operatorname{dist}(x, X)+\operatorname{dist}(x, Y) \geq C\left(\frac{\operatorname{dist}(x, X \cap Y)}{1+|x|^{2}}\right)^{\operatorname{deg} X \cdot \operatorname{deg} Y} \text { for } x \in \mathbb{C}^{N}
$$

A result similar to $\left(\mathrm{C}_{2}\right)$ was obtained by $\mathrm{Ji}$ et al. [20]. 
For real algebraic sets we have the following global Łojasiewicz inequality (see [22]). If $X, Y \subset \mathbb{R}^{N}$ are algebraic sets defined by systems of polynomial equations of degrees at most $d$, then for some positive constant $C$,

$$
\operatorname{dist}(x, X)+\operatorname{dist}(x, Y) \geq C\left(\frac{\operatorname{dist}(x, X \cap Y)}{1+|x|^{2}}\right)^{d(6 d-3)^{N-1}} \text { for } x \in \mathbb{R}^{N} .
$$

In particular, we have the following global Łojasiewicz inequality (see [22]). Let $F=\left(f_{1}, \ldots, f_{m}\right): \mathbb{R}^{N} \rightarrow \mathbb{R}^{m}$ be a polynomial mapping of degree $d$. Then for some positive constant $C$,

$$
|F(x)| \geq C\left(\frac{\operatorname{dist}\left(x, F^{-1}(0)\right)}{1+|x|^{2}}\right)^{d(6 d-3)^{N-1}} \text { for } x \in \mathbb{R}^{N}
$$

Moreover, if the set $F^{-1}(0)$ is compact, then

$$
\mathcal{L}_{\infty}^{\mathbb{R}}(F) \geq-d(6 d-3)^{N-1} .
$$

By using $\left(\mathrm{KS}_{4}\right)$ we obtain a global Łojasiewicz inequality for polynomial mappings.

Proposition 3.1 Let $X \subset \mathbb{R}^{N}$ be an algebraic set defined by a system of polynomial equations $g_{1}(x)=\cdots=g_{r}(x)=0$, where $g_{1}, \ldots, g_{r} \in \mathbb{R}\left[x_{1}, \ldots, x_{N}\right]$. Let $F$ : $\mathbb{R}^{N} \rightarrow \mathbb{R}^{m}$ be a polynomial mapping and let $d=\max \left\{\operatorname{deg} F, \operatorname{deg} g_{1}, \ldots, \operatorname{deg} g_{r}\right\}$. Then for some positive constant $C$,

$$
|F(x)| \geq C\left(\frac{\operatorname{dist}\left(x, F^{-1}(0) \cap X\right)}{1+|x|^{2}}\right)^{d(6 d-3)^{N-1}} \quad \text { for } x \in X .
$$

If, additionally, the set $X$ is unbounded and $F^{-1}(0) \cap X$ is compact, then

$$
\mathcal{L}_{\infty}^{\mathbb{R}}(F \mid X) \geq-d(6 d-3)^{N-1} .
$$

Indeed, let $G=\left(g_{1}, \ldots, g_{r}\right): \mathbb{R}^{N} \rightarrow \mathbb{R}^{r}$, and let $H: \mathbb{R}^{N} \rightarrow \mathbb{R}^{m+r}$ be a polynomial mapping defined by $H(x)=(F(x), G(x))$ for $x \in \mathbb{R}^{N}$. Then $H^{-1}(0)=$ $F^{-1}(0) \cap X$, so from $\left(\mathrm{KS}_{4}\right)$ we deduce the first assertion. If $F^{-1}(0) \cap X$ is compact, then so is $H^{-1}(0)$, and the second assertion follows immediately from the first (cf. $\left.\left(\mathrm{KS}_{5}\right)\right)$.

In the above proof we cannot apply $(\mathrm{Ch}),(\mathrm{K}),(\mathrm{CKT}),(\mathrm{J})$ or $\left(\mathrm{C}_{1}\right)$, because the complexification of a real polynomial mapping with compact real zero-set may have an unbounded zero-set.

The following global Łojasiewicz inequality for semialgebraic sets is the main result of this section. The proof is given in Sect. 4 .

Theorem 3.2 Let $X, Y \subset \mathbb{R}^{N}$ be closed semialgebraic sets. Set $r=r(X)+r(Y)$ and $d=\max \{\kappa(X), \kappa(Y)\}$. Then there exists a positive constant $C$ such that

$$
\operatorname{dist}(x, X)+\operatorname{dist}(x, Y) \geq C\left(\frac{\operatorname{dist}(x, X \cap Y)}{1+|x|^{d}}\right)^{d(6 d-3)^{N+r-1}} \text { for } x \in \mathbb{R}^{N} .
$$


Theorem 3.2 immediately implies the following.

Corollary 3.3 Let $F: X \rightarrow \mathbb{R}^{m}$ be a continuous semialgebraic mapping, where $X \subset \mathbb{R}^{N}$ is a closed semialgebraic set. If $d=\max \{\kappa(X), \kappa(Y)\}$ and $r=r(X)+r(Y)$, where $Y=\operatorname{graph} F$, then there exists a positive constant $C$ such that

$$
|F(x)| \geq C\left(\frac{\operatorname{dist}\left(x, F^{-1}(0) \cap X\right)}{1+|x|^{d}}\right)^{d(6 d-3)^{N+r-1}} \quad \text { for } x \in X .
$$

In particular, if the set $X$ is unbounded and $F^{-1}(0) \cap X$ is compact, then

$$
\mathcal{L}_{\infty}^{\mathbb{R}}(F \mid X) \geq(1-d) d(6 d-3)^{N+r-1}
$$

For a polynomial mapping $F: X \rightarrow \mathbb{R}^{m}$ we have $r(\operatorname{graph} F)=r(X)$ and $\kappa(\operatorname{graph} F)=\max \{\operatorname{deg} F, \kappa(X)\}$, so we obtain

Corollary 3.4 Let $F: X \rightarrow \mathbb{R}^{m}$ be a polynomial mapping, where $X \subset \mathbb{R}^{N}$ is a closed semialgebraic set. If $D=\max \{2, \kappa(X)\}$ and $d=\max \{\operatorname{deg} F, D\}$, and $r=2 r(X)$, then

$$
|F(x)| \geq C\left(\frac{\operatorname{dist}\left(x, F^{-1}(0) \cap X\right)}{1+|x|^{D}}\right)^{d(6 d-3)^{N+r-1}} \quad \text { for } x \in X
$$

In particular, if the set $X$ is unbounded and $F^{-1}(0) \cap X$ is compact, then

$$
\mathcal{L}_{\infty}^{\mathbb{R}}(F \mid X) \geq-\frac{D}{2} d(6 d-3)^{N+r-1}
$$

The above corollary is not a direct consequence of Corollary 3.3, so we will prove it separately in Sect. 4.

\section{Proofs of Theorems 2.1 and 3.2 and of Corollary 3.4}

It suffices to consider the case when $X$ and $Y$ are basic closed semialgebraic sets. So, let

$$
\begin{aligned}
& X=\left\{x \in \mathbb{R}^{N}: g_{1,1}(x) \geq 0, \ldots, g_{1, r(X)}(x) \geq 0, h_{1,1}(x)=\cdots=h_{1, l}(x)=0\right\}, \\
& Y=\left\{x \in \mathbb{R}^{N}: g_{2,1}(x) \geq 0, \ldots, g_{2, r(Y)}(x) \geq 0, h_{2,1}(x)=\cdots=h_{2, l}(x)=0\right\},
\end{aligned}
$$

where $g_{i, j}, h_{i, s} \in \mathbb{R}\left[x_{1}, \ldots, x_{N}\right]$. We may indeed assume that $X$ and $Y$ are defined by the same number of equations, because we can repeat the same equations if necessary. Let $r_{1}=r(X), r_{2}=r(Y), r=r_{1}+r_{2}$, and let $G_{i}: \mathbb{R}^{N} \times \mathbb{R}^{r} \rightarrow \mathbb{R}^{r_{i}}, i=1,2$, be the polynomial mappings defined by

$$
\begin{aligned}
& G_{1}\left(x, y_{1}, \ldots, y_{r}\right)=\left(g_{1,1}(x)-y_{1}^{2}, \ldots, g_{1, r_{1}}(x)-y_{r_{1}}^{2}\right), \\
& G_{2}\left(x, y_{1}, \ldots, y_{r}\right)=\left(g_{2,1}(x)-y_{r_{1}+1}^{2}, \ldots, g_{2, r_{2}}(x)-y_{r_{1}+r_{2}}^{2}\right) .
\end{aligned}
$$


Let

$$
\begin{aligned}
& A=\left\{\left(x, y_{1}, \ldots, y_{r}\right) \in \mathbb{R}^{N} \times \mathbb{R}^{r}: G_{1}(x, y)=0, h_{1,1}(x)=\cdots=h_{1, l}(x)=0\right\}, \\
& B=\left\{\left(x, y_{1}, \ldots, y_{r}\right) \in \mathbb{R}^{N} \times \mathbb{R}^{r}: G_{2}(x, y)=0, h_{2,1}(x)=\cdots=h_{2, l}(x)=0\right\} .
\end{aligned}
$$

Then the sets $A$ an $B$ are algebraic and $\pi(A)=X, \pi(B)=Y$, where $\pi: \mathbb{R}^{N} \times \mathbb{R}^{r} \ni$ $(x, y) \mapsto x \in \mathbb{R}^{N}$. Moreover, $\operatorname{deg} G_{1}, \operatorname{deg} G_{2} \leq d$, provided $d>1$.

From the definitions of $A$ and $B$, we immediately obtain

$$
\forall_{x_{1} \in X} \forall_{x_{2} \in Y} \exists_{y \in \mathbb{R}^{r}}\left(x_{1}, y\right) \in A \wedge\left(x_{2}, y\right) \in B
$$

moreover,

$$
\begin{gathered}
\forall_{x \in \mathbb{R}^{N} \backslash X} \exists_{x_{1} \in X} \forall_{y \in \mathbb{R}^{r}}\left[\operatorname{dist}(x, X)=\left|x-x_{1}\right| \wedge\left(x_{1}, y\right) \in A \Rightarrow\right. \\
\operatorname{dist}(x, X) \geq \operatorname{dist}((x, y), A)]
\end{gathered}
$$

and

$$
\begin{gathered}
\forall_{x \in \mathbb{R}^{N} \backslash Y} \exists_{x_{2} \in Y} \forall_{y \in \mathbb{R}^{r}}\left[\operatorname{dist}(x, Y)=\left|x-x_{2}\right| \wedge\left(x_{2}, y\right) \in B \Rightarrow\right. \\
\operatorname{dist}(x, Y) \geq \operatorname{dist}((x, y), B)] .
\end{gathered}
$$

Indeed, we will prove (4.2); the proof of (4.3) is similar. Take $x \in \mathbb{R}^{N} \backslash X$ and let $x_{1} \in X$ satisfy $\operatorname{dist}(x, X)=\left|x-x_{1}\right|$. So, for any $y \in \mathbb{R}^{r}$ such that $\left(x_{1}, y\right) \in A$, we have

$$
\operatorname{dist}(x, X)=\left|x-x_{1}\right|=\left|(x, y)-\left(x_{1}, y\right)\right| \geq \operatorname{dist}((x, y), A) .
$$

This gives (4.2).

Proof of Theorem 2.1 We will assume that the origin is a non-isolated point of $X \cap Y$; otherwise, we proceed in the same way using formula $(\mathrm{G})$ instead of $\left(\mathrm{KS}_{1}\right)$. Let $p=d(6 d-3)^{N+r-1}$.

Claim 1. The assertion (2.4) is equivalent to

$$
\operatorname{dist}(x, Y) \geq C^{\prime} \operatorname{dist}(x, X \cap Y)^{p} \quad \text { for } x \in(\partial X) \cap U_{1}
$$

for a neighbourhood $U_{1}=\left\{x \in \mathbb{R}^{N}:|x|<\rho\right\}$ of the origin, $\rho<1$, and some positive constant $C^{\prime}$, where $\partial X$ denotes the boundary of $X$ (cf. [9, Lem. 4.2] and [22, Proof of Theorem 2]). Indeed, the implication $(2.4) \Rightarrow(4.4)$ is obvious. Assume that the converse fails. Then for a neighbourhood $U_{2}=\left\{x \in \mathbb{R}^{N}:|x|<\frac{\rho}{2}\right\}$ of the origin, there exists a sequence $a_{v} \in U_{2}$ such that $a_{v} \rightarrow 0$ and

$$
\operatorname{dist}\left(a_{v}, X\right)+\operatorname{dist}\left(a_{v}, Y\right)<\frac{1}{v} \operatorname{dist}\left(a_{v}, X \cap Y\right)^{p} \quad \text { for } v \in \mathbb{N} \text {. }
$$


Taking a subsequence if necessary, it suffices to consider two cases: $a_{v} \notin X$ for $v \in \mathbb{N}$ or $a_{v} \in \operatorname{Int} X$ for $v \in \mathbb{N}$.

Assume that $a_{v} \notin X$ for $v \in \mathbb{N}$. Let $x_{v} \in(\partial X) \cap U_{1}$ be such that $\operatorname{dist}\left(a_{v}, X\right)=$ $\left|a_{v}-x_{v}\right|$. Since $\rho<1$, we have $\operatorname{dist}\left(a_{v}, X\right)^{\frac{1}{p}} \geq \operatorname{dist}\left(a_{v}, X\right)$. So, for some $C^{\prime \prime}>0$,

$$
\left[\operatorname{dist}\left(a_{v}, X\right)+\operatorname{dist}\left(a_{v}, Y\right)\right]^{\frac{1}{p}} \geq \operatorname{dist}\left(a_{v}, X\right)^{\frac{1}{p}} \geq C^{\prime \prime} \operatorname{dist}\left(a_{v}, X\right),
$$

and, by (4.4),

$$
\left[\operatorname{dist}\left(a_{v}, X\right)+\operatorname{dist}\left(a_{v}, Y\right)\right]^{\frac{1}{p}} \geq \operatorname{dist}\left(x_{v}, Y\right)^{\frac{1}{p}} \geq C^{\prime \prime} \operatorname{dist}\left(x_{v}, X \cap Y\right) .
$$

Since $\operatorname{dist}\left(a_{v}, X\right)+\operatorname{dist}\left(x_{v}, X \cap Y\right) \geq \operatorname{dist}\left(a_{v}, X \cap Y\right)$, by adding the above inequalities, we obtain

$$
\left[\operatorname{dist}\left(a_{\nu}, X\right)+\operatorname{dist}\left(a_{\nu}, Y\right)\right]^{\frac{1}{p}} \geq \frac{C^{\prime \prime}}{2} \operatorname{dist}\left(a_{\nu}, X \cap Y\right) .
$$

This contradicts (4.5) and proves the Claim in this case.

Now assume that all $a_{v}$ are in Int $X$. Let $y_{v} \in Y \cap U_{1}$ be $\operatorname{such}$ that $\operatorname{dist}\left(a_{v}, Y\right)=$ $\left|a_{v}-y_{v}\right|$. By (4.5) we see that $y_{v} \notin X$, so there exists $x_{v} \in(\partial X) \cap\left[a_{v}, y_{v}\right]$, where $\left[a_{v}, y_{v}\right]$ is the segment with endpoints $a_{v}, y_{v}$.

By (4.5) and the choice of $\rho$,

$$
\left|a_{v}-x_{v}\right| \leq \operatorname{dist}\left(a_{v}, Y\right)<\frac{1}{v} \operatorname{dist}\left(a_{v}, X \cap Y\right)^{p}<\frac{1}{2} \operatorname{dist}\left(a_{v}, X \cap Y\right) \text { for } v \geq 2 .
$$

Hence,

$$
\operatorname{dist}\left(x_{v}, X \cap Y\right) \geq \operatorname{dist}\left(a_{v}, X \cap Y\right)-\left|a_{v}-x_{v}\right| \geq \frac{1}{2} \operatorname{dist}\left(a_{v}, X \cap Y\right) \text { for } v \geq 2
$$

This together with (4.5) gives

$\operatorname{dist}\left(x_{v}, Y\right) \leq \operatorname{dist}\left(a_{v}, Y\right)<\frac{1}{v} \operatorname{dist}\left(a_{v}, X \cap Y\right)^{p} \leq \frac{2^{p}}{v} \operatorname{dist}\left(x_{v}, X \cap Y\right)^{p} \quad$ for $v \geq 2$.

This contradicts (4.4) and proves the claim in this case. Summing up, we have proved Claim 1.

If $d=1$, then the assertion is trivial. Assume that $d>1$. By $\left(\mathrm{KS}_{1}\right)$, there exists a positive constant $C$ such that

$$
\operatorname{dist}((x, y), A)+\operatorname{dist}((x, y), B) \geq C \operatorname{dist}((x, y), A \cap B)^{d(6 d-3)^{N+r-1}}
$$

in a neighbourhood $W$ of $0 \in \mathbb{R}^{N+r}$. Obviously, for any $(x, y) \in \mathbb{R}^{N+r}$,

$$
\operatorname{dist}((x, y), A \cap B) \geq \operatorname{dist}(x, X \cap Y) .
$$


One can assume that $g_{i, j}(0)=0$ for any $i, j$. Indeed, if $g_{i, j}(0)<0$ for some $i, j$, then $0 \notin X$ or $0 \notin Y$, which contradicts the assumption. If $g_{i, j}(0)>0$ for some $i, j$, then we can omit this inequality in the definition of $X$, respectively $Y$, and the germ at 0 of $X$, respectively $Y$ will not change. If $g_{i, j}(0)>0$ for any $i, j$, then the assertion reduces to $\left(\mathrm{KS}_{2}\right)$. So, there exists a neighbourhood $W_{1}=U_{3} \times U^{\prime} \times U^{\prime \prime} \subset W$ of $0 \in \mathbb{R}^{N+r}$, where $U_{3} \subset \mathbb{R}^{N}, U^{\prime} \subset \mathbb{R}^{r(X)}$ and $U^{\prime \prime} \subset \mathbb{R}^{r(Y)}$ such that:

$$
\begin{aligned}
\text { for any }\left(x_{1}, y^{\prime}, y^{\prime \prime}\right) \in \mathrm{A}, & \text { where } x_{1} \in \mathbb{R}^{N}, y^{\prime} \in \mathbb{R}^{r(X)}, y^{\prime \prime} \in \mathbb{R}^{r(Y)} \\
& \text { if } x_{1} \in X \cap U_{3} \text {, then } y^{\prime} \in U^{\prime}
\end{aligned}
$$

and

$$
\begin{aligned}
\text { for any }\left(x_{2}, y^{\prime}, y^{\prime \prime}\right) \in B, & \text { where } x_{2} \in \mathbb{R}^{N}, y^{\prime} \in \mathbb{R}^{r(X)}, y^{\prime \prime} \in \mathbb{R}^{r(Y)} \\
& \text { if } x_{2} \in Y \cap U_{3}, \text { then } y^{\prime \prime} \in U^{\prime \prime} .
\end{aligned}
$$

Let $U \subset U_{3}$ be a neighbourhood of $0 \in \mathbb{R}^{N}$. If $(\partial X) \cap U=\emptyset$, then $U \subset X$ and the assertion is obvious. Assume that $(\partial X) \cap U \neq \emptyset$. Take $x \in(\partial X) \cap U$, and let $x^{\prime} \in Y$ be a point for which $\operatorname{dist}(x, Y)=\left|x-x^{\prime}\right|$. By (4.1) there exists $y \in \mathbb{R}^{r}$ such that $(x, y) \in A$ and $\left(x^{\prime}, y\right) \in B$. Diminishing the neighbourhood $U$ if necessary, we may assume that $x^{\prime} \in U_{3}$. By (4.8) and (4.9) we see that $(x, y) \in W$, so, by (4.2) and (4.3),

$$
\operatorname{dist}(x, Y) \geq \operatorname{dist}((x, y), A)+\operatorname{dist}((x, y), B) .
$$

Summing up, (4.6), (4.7) and Claim 1 give the assertion.

Proof of Theorem 3.2 Let $p=d(6 d-3)^{N+r-1}$. If $d=1$ then the assertion is trivial. If $X \backslash Y=\emptyset$ or $Y \backslash X=\emptyset$, then the assertion is obvious. So, we will assume that $X \backslash Y \neq \emptyset, Y \backslash X \neq \emptyset$ and $d>1$. In particular $\partial X \neq \emptyset$.

By $\left(\mathrm{KS}_{3}\right)$ we have

$$
\operatorname{dist}((x, y), A)+\operatorname{dist}((x, y), B) \geq C\left(\frac{\operatorname{dist}((x, y), A \cap B)}{1+|(x, y)|^{2}}\right)^{p}
$$

for $(x, y) \in \mathbb{R}^{N+r}$. Since $\operatorname{dist}((x, y), A \cap B) \geq \operatorname{dist}(x, X \cap Y)$ for any $(x, y) \in \mathbb{R}^{N+r}$ (see (4.7)), the inequality (4.10) gives

$$
\operatorname{dist}((x, y), A)+\operatorname{dist}((x, y), B) \geq C\left(\frac{\operatorname{dist}(x, X \cap Y)}{1+|(x, y)|^{2}}\right)^{p}
$$

for $(x, y) \in \mathbb{R}^{N+r}$.

Claim 2. The assertion (3.1) is equivalent to

$$
\operatorname{dist}(x, Y) \geq C^{\prime}\left(\frac{\operatorname{dist}(x, X \cap Y)}{1+|x|^{d}}\right)^{p} \text { for } x \in \partial X
$$


for some positive constant $C^{\prime}$ (cf. [9, Lem. 4.2] and [22, Proof of Theorem 2]). Indeed, the implication $(3.1) \Rightarrow(4.12)$ is obvious. Assume that the converse fails. Then there exists a sequence $a_{v} \in \mathbb{R}^{N}$ such that

$$
\operatorname{dist}\left(a_{v}, X\right)+\operatorname{dist}\left(a_{v}, Y\right)<\frac{1}{v}\left(\frac{\operatorname{dist}\left(a_{v}, X \cap Y\right)}{1+\left|a_{v}\right|^{d}}\right)^{p} \quad \text { for } v \in \mathbb{N} .
$$

By using Theorem 2.1 we see that $\left|a_{v}\right| \rightarrow \infty$. Taking subsequences of $a_{v}$ if necessary, it suffices to consider two cases: $a_{v} \notin X$ for $v \in \mathbb{N}$ or $a_{v} \in \operatorname{Int} X$ for $v \in \mathbb{N}$.

Suppose $a_{v} \notin X$ for $v \in \mathbb{N}$. Let $b_{v} \in \partial X$ be such that $\operatorname{dist}\left(a_{v}, X\right)=\left|a_{v}-b_{v}\right|$. Since $\left(\frac{\operatorname{dist}\left(a_{\nu}, X \cap Y\right)}{1+\left|a_{v}\right|^{d}}\right)^{p}$ is a bounded sequence, we have $\left|b_{v}-a_{v}\right|=\operatorname{dist}\left(a_{v}, X\right) \rightarrow 0$. So, for some $C^{\prime \prime}>0$ and sufficiently large $v$,

$$
\left[\operatorname{dist}\left(a_{v}, X\right)+\operatorname{dist}\left(a_{v}, Y\right)\right]^{\frac{1}{p}} \geq \operatorname{dist}\left(a_{v}, X\right)^{\frac{1}{p}} \geq C^{\prime \prime}\left(\frac{\operatorname{dist}\left(a_{v}, X\right)}{1+\left|a_{v}\right|^{d}}\right),
$$

and, by (4.12) and the fact that $\left|a_{v}\right| \rightarrow \infty$ and $\left|b_{v}-a_{v}\right| \rightarrow 0$,

$$
\left[\operatorname{dist}\left(a_{v}, X\right)+\operatorname{dist}\left(a_{v}, Y\right)\right]^{\frac{1}{p}} \geq \operatorname{dist}\left(b_{v}, Y\right)^{\frac{1}{p}} \geq C^{\prime \prime}\left(\frac{\operatorname{dist}\left(b_{v}, X \cap Y\right)}{1+\left|a_{v}\right|^{d}}\right)
$$

Since $\operatorname{dist}\left(a_{\nu}, X\right)+\operatorname{dist}\left(b_{v}, X \cap Y\right) \geq \operatorname{dist}\left(a_{\nu}, X \cap Y\right)$, by adding the above inequalities we obtain

$$
\left[\operatorname{dist}\left(a_{\nu}, X\right)+\operatorname{dist}\left(a_{v}, Y\right)\right]^{\frac{1}{p}} \geq \frac{C^{\prime \prime}}{2}\left(\frac{\operatorname{dist}\left(a_{\nu}, X \cap Y\right)}{1+\left|a_{\nu}\right|^{d}}\right) .
$$

This contradicts (4.13) and proves the claim in this case.

Consider now the case $a_{v} \in \operatorname{Int} X$ for $v \in \mathbb{N}$. Let $y_{v} \in Y$ be such that $\operatorname{dist}\left(a_{v}, Y\right)=$ $\left|a_{v}-y_{v}\right|$. By (4.13) we see that $y_{v} \notin X$, so there exist $x_{v} \in(\partial X) \cap\left[a_{v}, y_{v}\right]$ for $v \in \mathbb{N}$. By (4.13), for sufficiently large $v$,

$$
\left|a_{v}-x_{v}\right| \leq \operatorname{dist}\left(a_{v}, Y\right)<\frac{1}{v}\left(\frac{\operatorname{dist}\left(a_{v}, X \cap Y\right)}{1+\left|a_{v}\right|^{d}}\right)^{p}<\frac{1}{2} \operatorname{dist}\left(a_{v}, X \cap Y\right) .
$$

Hence,

$$
\operatorname{dist}\left(x_{v}, X \cap Y\right) \geq \operatorname{dist}\left(a_{v}, X \cap Y\right)-\left|a_{v}-x_{v}\right| \geq \frac{1}{2} \operatorname{dist}\left(a_{v}, X \cap Y\right) .
$$

This together with (4.13) gives

$$
\operatorname{dist}\left(x_{v}, Y\right) \leq \operatorname{dist}\left(a_{v}, Y\right)<\frac{1}{v}\left(\frac{\operatorname{dist}\left(a_{v}, X \cap Y\right)}{1+\left|a_{v}\right|^{d}}\right)^{p} \leq \frac{2^{p}}{v}\left(\frac{\operatorname{dist}\left(x_{v}, X \cap Y\right)}{1+\left|a_{v}\right|^{d}}\right)^{p}
$$


By (4.14), for sufficiently large $v$ we have $\left|x_{v}\right| \leq 2\left|a_{v}\right|$, so, for a positive constant $C^{\prime \prime \prime}$,

$$
\operatorname{dist}\left(x_{v}, Y\right) \leq \frac{C^{\prime \prime \prime}}{v}\left(\frac{\operatorname{dist}\left(x_{v}, X \cap Y\right)}{1+\left|x_{v}\right|^{d}}\right)^{p}
$$

This contradicts (4.12) and proves the claim in this case. Summing up, we have proved Claim 2.

Take any $x_{0} \in \partial X$. By (4.1), (4.2) and (4.3) there exist $x_{2} \in Y$ and $y_{0} \in \mathbb{R}^{r}$ such that $\left(x_{0}, y_{0}\right) \in A,\left(x_{2}, y_{0}\right) \in B$, and $\operatorname{dist}\left(x_{0}, Y\right)=\left|x_{0}-x_{2}\right| \geq \operatorname{dist}\left(\left(x_{0}, y_{0}\right), B\right)$. Hence from (4.11),

$$
\operatorname{dist}\left(x_{0}, Y\right) \geq C\left(\frac{\operatorname{dist}\left(x_{0}, X \cap Y\right)}{1+\left|\left(x_{0}, y_{0}\right)\right|^{2}}\right)^{p} .
$$

It is easy to observe that there exist constants $C_{1}, R_{1}>0$ such that for $(x, y) \in A$, $|(x, y)| \geq R_{1}$ we have $C_{1}|y|^{2} \leq|x|^{d}$. Since $d \geq 2$, for a constant $C_{2}>0$ we obtain $|(x, y)| \leq C_{2}|x|^{d / 2}$ for $(x, y) \in A,|(x, y)| \geq R_{1}$. Hence from (4.15) we easily deduce

$$
\operatorname{dist}\left(x_{0}, Y\right) \geq C\left(\frac{\operatorname{dist}\left(x_{0}, X \cap Y\right)}{1+C_{2}^{2}\left|x_{0}\right|^{d}}\right)^{p},
$$

provided $\left|x_{0}\right| \geq R_{1}$. So, diminishing $C$ if necessary, we obtain (4.16) for all $x_{0} \in \partial X$. This together with Claim 2 gives the assertion of Theorem 3.3.

Proof of Corollary 3.4 Let $H: \mathbb{R}^{N+r} \rightarrow \mathbb{R}^{m+r+l}$ be a polynomial mapping defined by

$$
H(x, y)=\left(F(x), G_{1}(x, y), h_{1,1}(x), \ldots, h_{1, l}(x)\right), \quad x \in \mathbb{R}^{N}, y \in \mathbb{R}^{r} .
$$

Then $\operatorname{deg} H \leq d$. Let $V=F^{-1}(0) \cap X$ and let $Z=H^{-1}(0)$. By $\left(\mathrm{KS}_{4}\right)$, for some positive constant $C$ we have

$$
|H(x, y)| \geq C\left(\frac{\operatorname{dist}((x, y), Z)}{1+|(x, y)|^{2}}\right)^{d(6 d-3)^{N+r-1}} \text { for } \quad(x, y) \in \mathbb{R}^{N} \times \mathbb{R}^{r}
$$

Because $\operatorname{dist}((x, y), Z) \geq \operatorname{dist}(x, V)$, we obtain

$$
|H(x, y)| \geq C\left(\frac{\operatorname{dist}(x, V)}{1+|(x, y)|^{2}}\right)^{d(6 d-3)^{N+r-1}} \text { for }(x, y) \in \mathbb{R}^{N} \times \mathbb{R}^{r} .
$$

It is easy to observe that there exist constants $C_{1}, R_{1}>0$ such that for $(x, y) \in A$ with $|(x, y)| \geq R_{1}$ we have $C_{1}|y|^{2} \leq|x|^{D}$. Since $D \geq 2$, for a constant $C_{2}>0$ we obtain $|(x, y)| \leq C_{2}|x|^{D / 2}$ for $(x, y) \in A,|(x, y)| \geq R_{1}$. Hence from (4.17) we easily deduce (3.2) for $x \in X,|x| \geq R_{1}$. So, diminishing $C$ if necessary, we obtain (3.2) for all $x \in X$. 
We now show the second assertion of the corollary. Since $X$ is unbounded, we may assume that so is $A$. Since $V$ is compact, so is $H^{-1}(0)$. By $\left(\mathrm{KS}_{5}\right)$ we have $\mathcal{L}_{\infty}(H) \geq-d(6 d-3)^{N+r-1}$, in particular for some constants $C, R>0$,

$$
|H(x, y)| \geq C|(x, y)|^{-d(6 d-3)^{N+r-1}} \text { for }(x, y) \in A, \quad|(x, y)| \geq R .
$$

Since $|(x, y)| \leq C_{2}|x|^{D / 2}$ for $(x, y) \in A,|(x, y)| \geq R_{1}$, it follows that, for some constant $C_{3}>0$.

$$
|F(x)|=|H(x, y)| \geq C_{3}|x|^{-\frac{D}{2} d(6 d-3)^{N+r-1}} \quad \text { for }(x, y) \in A,|(x, y)| \geq R,
$$

and $\mathcal{L}_{\infty}^{\mathbb{R}}(F \mid X) \geq-\frac{D}{2} d(6 d-3)^{N+r-1}$. This ends the proof of Corollary 3.4.

Acknowledgments This research was partially supported by OPUS Grant No. 2012/07/B/ST1/03293 (Poland) and ANR project STAAVF (France).

Open Access This article is distributed under the terms of the Creative Commons Attribution 4.0 International License (http://creativecommons.org/licenses/by/4.0/), which permits unrestricted use, distribution, and reproduction in any medium, provided you give appropriate credit to the original author(s) and the source, provide a link to the Creative Commons license, and indicate if changes were made.

\section{References}

1. Achilles, R., Tworzewski, P., Winiarski, T.: On improper isolated intersection in complex analytic geometry. Ann. Polon. Math. 51, 21-36 (1990)

2. Basu, S., Pollack, R., Roy, M.-F.: Algorithms in Real Algebraic Geometry. Algorithms and Computation in Mathematics, vol. 10, 2nd edn. Springer, Berlin (2006)

3. Bochnak, J., Risler, J.J.: Sur les exposants de Łojasiewicz. Comment. Math. Helv. 50, 493-507 (1975)

4. Bochnak, J., Coste, M., Roy, M.-F.: Real Algebraic Geometry. Springer, Berlin (1998)

5. Bröcker, L.: Minimale Erzeugung von Positivbereichen. Geom. Dedic. 16(3), 335-350 (1984)

6. Bröcker, L.: On basic semialgebraic sets. Expos. Math. 9(4), 289-334 (1991)

7. Chạdzyński, J.: On proper polynomial mappings. Bull. Polish Acad. Sci. Math. 31, 115-120 (1983)

8. Cygan, E.: Intersection theory and separation exponent in complex analytic geometry. Ann. Polon. Math. 69, 287-299 (1998)

9. Cygan, E.: A note on separation of algebraic sets and the Łojasiewicz exponent for polynomial mappings. Bull. Sci. Math. 129(2), 139-147 (2005)

10. Cygan, E., Krasiński, T., Tworzewski, P.: Separation of algebraic sets and the Łojasiewicz exponent of polynomial mappings. Invent. Math. 136(1), 75-87 (1999)

11. D'Acunto, D., Kurdyka, K.: Explicit bounds for the Łojasiewicz exponent in the gradient inequality for polynomials. Ann. Polon. Math. 87, 51-61 (2005)

12. van den Dries, L., Miller, C.: Geometric categories and o-minimal structures. Duke Math. J. 84, $497-$ 540 (1996)

13. Gabrielov, A.: Multiplicities of Pfaffian intersection, and the Łojasiewicz inequality. Sel. Math. (N.S.) 1, 113-127 (1995)

14. Gwoździewicz, J.: The Łojasiewicz exponent of an analytic function at an isolated zero. Comment. Math. Helv. 74, 364-375 (1999)

15. Heintz, J., Roy, M.-F., Solernó, P.: Sur la complexité du principe de Tarski-Seidenberg. Bull. Soc. Math. Fr. 118, 101-126 (1990)

16. Hironaka, H.: Subanalytic Sets. In: Number Theory, Algebraic Geometry and Commutative Algebra (Kinokuniya, Tokyo). Volume in Honor of Yasuo Akizuki, pp. 453-493 (1973)

17. Hörmander, L.: On the division of distributions by polynomials. Ark. Mat. 3, 555-568 (1958)

18. Jelonek, Z.: On the effective Nullstellensatz. Invent. Math. 162(1), 1-17 (2005)

19. Jelonek, Z.: On the Łojasiewicz exponent. Hokkaido Math. J. 35(2), 471-485 (2006) 
20. Ji, S., Kollár, J., Shiffman, B.: A global Łojasiewicz inequality for algebraic varieties. Trans. Am. Math. Soc. 329, 813-818 (1992)

21. Kollár, J.: Sharp effective Nullstellensatz. J. Am. Math. Soc. 1, 963-975 (1988)

22. Kurdyka, K., Spodzieja, S.: Separation of real algebraic sets and the Łojasiewicz exponent. Proc. Am. Math. Soc 142(9), 3089-3102 (2014)

23. Kurdyka, K., Spodzieja, S.: Convexifying positive polynomials and sums of squares approximation. SIAM J. Optim. 25(4), 2512-2536 (2015)

24. Kurdyka, K., Mostowski, T., Parusiński, A.: Proof of the gradient conjecture of R. Thom. Ann. Math (2) 152(3), 763-792 (2000)

25. Łojasiewicz, S.: Division d'une distribution par une fonction analytique de variables réelles. C. R. Acad. Sci. Paris 246, 683-686 (1958)

26. Łojasiewicz, S.: Sur le problème de la division. Studia Math. 18, 81-136 (1959)

27. Li, G., Mordukhovich, B.S., Pham, T.S.: New fractional error bounds for polynomial systems with applications to Hölderian stability in optimization and spectral theory of tensors. Math. Progr. Ser. A 153(2), 333-362 (2015)

28. Niederman, L.: Hamiltonian stability and subanalytic geometry. Ann. Inst. Fourier (Grenoble) 56(3), 795-813 (2006)

29. Pham, T.S.: An explicit bound for the Łojasiewicz exponent of real polynomials. Kodai Math. J. 35(2), 311-319 (2012)

30. Płoski, A.: Multiplicity and the Łojasiewicz exponent. Banach Center Publications 20, Warsaw (1988)

31. Rodak, T., Spodzieja, S.: Effective formulas for the Łojasiewicz exponent at infinity. J. Pure Appl. Algebra 213, 1816-1822 (2009)

32. Rodak, T., Spodzieja, S.: Effective formulas for the local Łojasiewicz exponent. Math. Z. 268, 37-44 (2011)

33. Rodak, T., Spodzieja, S.: Equivalence of mappings at infinity. Bull. Sci. Math. 136(6), 679-686 (2012)

34. Roy, M.-F., Vorobjov, N.: The complexification and degree of a semi-algebraic set. Math. Z. 239(1), 131-142 (2002)

35. Scheiderer, C.: Stability index of real varieties. Invent. Math. 97(3), 467-483 (1989)

36. Schweighofer, M.: On the complexity of Schmüdgen's Positivstellensatz. J. Complex. 20, 529-543 (2004)

37. Schweighofer, M.: Optimization of polynomials on compact semialgebraic sets. SIAM J. Optim. 15(3), 805-825 (2005)

38. Simon, L.: Asymptotics for a class of nonlinear evolution equations, with applications to geometric problems. Ann. Math. (2) 118(3), 525-571 (1983)

39. Solernó, P.: Effective Łojasiewicz inequalities in semialgebraic geometry. Appl. Algebra Eng. Commun. Comput. 2(1), 2-14 (1991)

40. Spodzieja, S.: Multiplicity and the Łojasiewicz exponent. Ann. Polon. Math. 73, 257-267 (2000)

41. Spodzieja, S.: The Łojasiewicz exponent of subanalytic sets. Ann. Polon. Math. 87, 247-263 (2005)

42. Spodzieja, S., Szlachcińska, A.: Łojasiewicz exponent of overdetermined mappings. Bull. Polish Acad. Sci. Math. 61(1), 27-34 (2013)

43. Teissier, B.: Variétés polaires. I. Invariants polaires des singularités d'hypersurfaces. Invent. Math. 40(3), 267-292 (1977)

44. Tworzewski, P.: Intersection theory in complex analytic geometry. Ann. Polon. Math. 62, 177-191 (1995) 